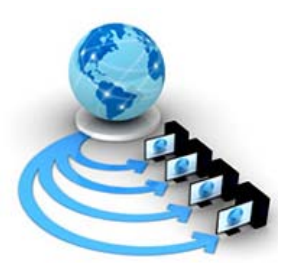

Volume 8, No. 9, November-December 2017

International Journal of Advanced Research in Computer Science

RESEARCH PAPER

\author{
Available Online at www.ijarcs.info
}

\title{
INVERSE SIGNED DOMINATING FUNCTIONS OF CORONA AND ROOTED PRODUCT GRAPHS
}

\author{
C. Shobha Rani \\ Department of Mathematics, \\ Madanapalle Institute of Technology \& Science, \\ Madanapalle-517325, India
}

\author{
S. Jeelani Begum \\ Department of Mathematics, \\ Madanapalle Institute of Technology \& Science, \\ Madanapalle-517325, India
}

\author{
G. S. S. Raju \\ Department of Mathematics, \\ JNTUA College of Engineering, \\ Pulivendula- 516390, India
}

\begin{abstract}
Graph theory is an interesting subject in mathematics. Applications in many fields like Linguistics, Engineering communications, Physical Sciences, Coding theory, Computer networking and Logical Algebra. The theory of domination in graphs has a wide range of applications. Among these applications, the most often discussed is a coding theory and communication networks. Inverse domination theory of graphs which are the important branches of graph theory. In this paper, we study the maximal inverse signed dominating functions of corona product graph of a path with a complete graph and rooted product graph of a path with a cycle.
\end{abstract}

Keywords: inverse signed dominating functions, inverse signed domination number, corona product graph, rooted product graph.

\section{INTRODUCTION}

Mostly Product of graphs occurs in discrete mathematics. In 1970, Frucht \& Harary [6] introduced a new product on two graphs $\mathrm{G}_{1}$ and $\mathrm{G}_{2}$, called corona product denoted by $G_{1} \square G_{2}$. The corona product of a path $P_{n}$ with a complete graph $K_{m}$ is a graph obtained by taking one copy of nvertex path $P_{n}$ and $\mathrm{n}$ copies of $K_{m}$ and then joining the $\mathrm{i}^{\text {th }}$ vertex of $P_{n}$ to every vertex of $\mathrm{i}^{\text {th }}$ copy of $K_{m}$ and it is denoted by $P_{\mathrm{n}} \square K_{m}$, where $\mathrm{n}>0$ and $\mathrm{m}>0$. In 1978, Godsil and McKay [1] introduced a new product on two graphs $G_{1}$ and $G_{2}$, called rooted product denoted by $G_{1} \circ G_{2}$. In this paper we consider the rooted product graph like, here $P_{\mathrm{n}}$ be a Path graph with n vertices and $C_{m}(m \geq 3)$ be a cycle with a sequence of $n$ rooted graphs $C_{m 1}, C_{m 2}, C_{m 3},---, C_{m n}$. Then by $P_{\mathrm{n}}\left(C_{m}\right)$ we denote the graph obtained by identifying the root of $C_{m i}$ with the $\mathrm{i}^{\text {th }}$ vertex of $P_{\mathrm{n}}$. We call $P_{\mathrm{n}}\left(C_{m}\right)$ the rooted product of $P_{\mathrm{n}}$ by $C_{m}$ and it is denoted by $P_{n} \circ C_{m}$. Every $\mathrm{i}^{\text {th }}$ vertex of $P_{\mathrm{n}}$ is merging with any one vertex in every $\mathrm{i}^{\text {th }}$ copy of $C_{m}$. So in $\mathrm{G}=P_{\mathrm{n}} \circ C_{m}, P_{\mathrm{n}}$ contains $\mathrm{n}$ vertices and $C_{m}$ contains (m-1) vertices in each copy of $C_{m}$.

In 1995, Dunbar, Hedetniemi, Henning and Slater [4] have studied about "Signed Domination in Graphs". Further we studied about signed domination in [2, 7]. In 1996, Favaron [5] have studied about "Signed domination in regular graphs”. In 2010, Zhong-sheng [3] have studied about “On Inverse Signed Total Domination in Graphs”. By using signed domination related parameters we can find out inverse signed domination parameters on product graphs.

\section{RESULTS ON ROOTED PRODUCT GRAPH}

Theorem 2.1: If $\mathrm{m}$ is divisible by 3 then the function $f: V \rightarrow\{-1,+1\}$ is defined by

$f(v)= \begin{cases}+1, & \text { if } m \equiv 1(\bmod 3) \text { vertices in each copy of } C_{m} \text { in } G, \\ -1, & \text { otherwise. }\end{cases}$

is a maximal inverse signed dominating function of a graph $\mathrm{G}=P_{\mathrm{n}} \circ C_{m}$ and inverse signed domination number of

$\mathrm{G}$ is $\gamma_{s}^{0}(G)=\left(\frac{-m n}{3}\right)$.

Proof: Consider the graph $\mathrm{G}=P_{\mathrm{n}} \circ C_{m}$ with $|V|$ number of vertices and $|E|$ number of edges.

Let $f$ be a function defined in the hypothesis. Suppose $\mathrm{m}$ is divisible by 3 .

Here +1 is assigned to $\left(\frac{m}{3}\right)$ vertices in each copy of $C_{m}$ in $\mathrm{G},-1$ is assigned to all other vertices in $\mathrm{G}$.

Case 1: Suppose $\mathrm{v} \in P_{\mathrm{n}}$ be such that

(i) As $\mathrm{d}(\mathrm{v})=4$ in $G$ then

$\sum_{u \in N[v]} f(u)=[(+1)+(-1)]+[(-1)+(-1)+(-1)]=-3$.

(ii) As $d(v)=3$ in $G$ then

$\sum_{u \in N[v]} f(u)=[(+1)+(-1)]+[(-1)+(-1)]=-2$.

Case 2: Suppose $\mathrm{v} \in C_{m}$ be such that $\mathrm{d}(\mathrm{v})=2$ in $G$ then $f(v)=-1, f(v)=+1$. 
(i) Then $\mathrm{N}[\mathrm{v}]$ contains 2 vertices of $C_{m}$ and one vertex of $P_{\mathrm{n}}$ in $G$.

If $f(v)=-1$ then $\sum_{u \in N[v]} f(u)=(-1)+[(-1)+(+1)]=-1$.

If $f(v)=+1$ then $\sum_{u \in N[v]} f(u)=(+1)+[(-1)+(-1)]=-1$.

(ii) Then $\mathrm{N}[\mathrm{v}]$ contains 3 vertices of $C_{m}$ and zero vertex of $P_{\mathrm{n}}$ in $G$.

If $f(v)=-1$ then $\sum_{u \in N[v]} f(u)=(-1)+[(-1)+(+1)]=-1$.

If $f(v)=+1$ then $\sum_{u \in N[v]} f(u)=(+1)+[(-1)+(-1)]=-1$.

From the above cases the function $f$ is an inverse signed dominating function, because $\sum_{u \in N[v]} f(u)<0, \forall v \in V$.

Now the maximality check for $f$, define $\mathrm{g}: \mathrm{V} \rightarrow\{-1,+1\}$ by $g(v)=\left\{\begin{array}{l}+1, \text { if any one vertex } v=u_{i} \in P_{n} \text { in } G, \\ +1, \text { if }\left(\frac{m}{3}\right) \text { vertices in each copy of } C_{m} \text { in } G, \\ -1, \quad \text { otherwise. }\end{array}\right.$

Here two cases are followed.

Case 3: Suppose $v \in P_{\mathrm{n}}$ be such that

(i)As $\mathrm{d}(\mathrm{v})=4$ in $G$, then $\mathrm{N}[\mathrm{v}]$ contains 2 vertices of $C_{m}$ and three vertices of $P_{\mathrm{n}}$ in $G$.

Sub case 1: Let $u_{\mathrm{i}} \in P_{n}$ ini ${ }^{\text {th }}$ copy of $\mathrm{G}$ then

$\sum_{u \in N[v]} g(u)=[(+1)+(-1)]+[(-1)+(-1)+(+1)]=-1$.

Sub case 2: Let $u_{\mathrm{i}} \notin P_{n}$ in $\mathrm{i}^{\text {th }}$ copy of $\mathrm{G}$ then

$\sum_{u \in N[v]} g(u)=[(+1)+(-1)]+[(-1)+(-1)+(-1)]=-3$.

(ii)As $\mathrm{d}(\mathrm{v})=3$ in $G$, then $\mathrm{N}[\mathrm{v}]$ contains 2 vertices of $C_{m}$ and two vertices of $P_{\mathrm{n}}$ in $G$.

Sub case 1: Let $u_{\mathrm{i}} \in P_{n}$ in $^{\text {th }}$ copy of $\mathrm{G}$ then

$\sum_{u \in N[v]} g(u)=[(+1)+(-1)]+[(-1)+(+1)]=0$.

Sub case 2: Let $u_{\mathrm{i}} \notin P_{n}$ in $\mathrm{i}^{\text {th }}$ copy of $\mathrm{G}$ then

$\sum_{u \in N[v]} g(u)=[(+1)+(-1)]+[(-1)+(-1)]=-2$.

Case 4: Suppose $\mathrm{v} \in C_{m}$ be such that $\mathrm{d}(\mathrm{v})=2$ in $G$,

(i)Here $\mathrm{N}[\mathrm{v}]$ contains 2 vertices of $C_{m}$ and one vertex of $P_{\mathrm{n}}$ in $G$ then $g(v)=-1$ or $g(v)=+1$.

Sub case 1 : Let $u_{\mathrm{i}} \in P_{n}$ in $\mathrm{i}^{\text {th }}$ copy of $\mathrm{G}$.

If $g(v)=-1$ then $\sum_{u \in N[v]} g(u)=(-1)+[(+1)+(+1)]=+1(>0)$.
If

$g(v)=+1$ then $\sum_{u \in N[v]} g(u)=(+1)+[(-1)+(+1)]=+1(>0)$.

Sub case 2: Let $u_{\mathrm{i}} \notin P_{n}$ in $\mathrm{i}^{\text {th }}$ copy of $\mathrm{G}$.

If $g(v)=-1$ then $\sum_{u \in N[v]} g(u)=(-1)+[(+1)+(-1)]=-1$.

If $g(v)=+1$ then $\sum_{u \in N[v]} g(u)=(+1)+[(-1)+(-1)]=-1$.

(ii)Here $\mathrm{N}[\mathrm{v}]$ contains 3 vertices of $C_{m}$ and zero vertex of $P_{\mathrm{n}}$ in $G$.

If $g(v)=-1$ then $\sum_{u \in N[v]} g(u)=(-1)+[(+1)+(-1)]=-1$.

If $g(v)=+1$ then $\sum_{u \in N[v]} g(u)=(+1)+[(-1)+(-1)]=-1$.

From the above cases, we get $\sum_{u \in N[v]} g(u)>0$, for some $v \in V$.

This implies that the function $g$ is not an inverse signed dominating function. Hence $f$ is a maximal inverse signed dominating function on G. Now inverse signed total domination number is the sum of the function value of all vertices in $\mathrm{G}$, that is

$$
\sum_{u \in V(G)} f(u)=(\underbrace{\frac{m}{3}(+1)}_{n-\text { times }})+\underbrace{\left((m)-\frac{m}{3}\right)(-1)}_{n \text {-times }}=-m n+\frac{2 m n}{3}=\frac{-m n}{3} . .
$$

Therefore $\gamma_{s}^{0}(G)=\left(\frac{-m n}{3}\right)$.

Theorem 2.2: If $m=3 k+1$ or $3 k+2$ is not divisible by 3 then inverse signed domination number of $G$ is $\gamma_{s}^{0}(G)= \begin{cases}n\left[2\left[\frac{m}{3}\right\rfloor-m\right], & \text { if } m=3 k+1 . \\ \left.n\left[2 \mid \frac{m}{3}\right\rceil-m\right], & \text { if } m=3 k+2 .\end{cases}$

Proof: Consider the graph $\mathrm{G}=P_{\mathrm{n}} \circ C_{m}$ with $|V|$ number of vertices and $|E|$ number of edges.

Case I: Suppose $\mathrm{m}=3 \mathrm{k}+1$

Let $f: V \rightarrow\{-1,+1\}$ be a function defined by $f(v)=\left\{\begin{array}{l}+1, \text { if } m \equiv 1(\bmod 3) \text { vertices in each copy of } C_{m} \text { in } G, \\ -1, \quad \text { otherwise. }\end{array}\right.$ Here +1 is assigned to $\left\lfloor\frac{m}{3}\right\rfloor$ vertices in each copy of $C_{m}$ in $\mathrm{G},-1$ is assigned to all other vertices in $\mathrm{G}$.

Case 1: Suppose $\mathrm{v} \in P_{\mathrm{n}}$ be such that

(i) As $\mathrm{d}(\mathrm{v})=4$ in $G$ then

$\sum_{u \in N[v]} f(u)=[(+1)+(-1)]+[(-1)+(-1)+(-1)]=-3$.

(ii) As $\mathrm{d}(\mathrm{v})=3$ in $G$ then

$\sum_{u \in N[v]} f(u)=[(+1)+(-1)]+[(-1)+(-1)]=-2$. 
Case 2: Suppose $\mathrm{v} \in C_{m}$ be such that $\mathrm{d}(\mathrm{v})=2$ in $G$ then $f(v)=-1, f(v)=+1$.

(i) Here $\mathrm{N}[\mathrm{v}]$ contains 2 vertices of $C_{m}$ and one vertex of $P_{\mathrm{n}}$ in $G$.

If $f(v)=-1$ then $\sum_{u \in N[v]} f(u)=(-1)+[(-1)+(-1)]=-3$.

If $f(v)=+1$ then $\sum_{u \in N[v]} f(u)=(+1)+[(-1)+(-1)]=-1$.

(ii) Here $\mathrm{N}[\mathrm{v}]$ contains 3 vertices of $C_{m}$ and zero vertex of $P_{\mathrm{n}}$ in $G$.

If $f(v)= \pm 1$ then $\sum_{u \in N[v]} f(u)=(-1)+[(-1)+(+1)]=-1$.

From the above cases the function $f$ is an inverse signed dominating function, because $\sum_{u \in N[v]} f(u)<0, \forall v \in V$.

Now the maximality check for $f$, define $\mathrm{g}: \mathrm{V} \rightarrow\{-1,+1\}$ by $g(v)=\left\{\begin{array}{l}+1, \text { if any one vertex } v=u_{i} \in P_{n} \text { in } G, \\ +1, \text { if }\left\lfloor\frac{m}{3}\right\rfloor \text { vertices in each copy of } C_{m} \text { in } G, \\ -1, \quad \text { otherwise. }\end{array}\right.$

Here two cases are followed.

Case 3: Suppose $\mathrm{v} \in P_{\mathrm{n}}$ be such that

(i)As $\mathrm{d}(\mathrm{v})=4$ in $G$, then $\mathrm{N}[\mathrm{v}]$ contains 2 vertices of $C_{m}$ and three vertices of $P_{\mathrm{n}}$ in $G$.

Sub case 1 : Let $u_{\mathrm{i}} \in P_{n}$ ini $^{\text {th }}$ copyof $\mathrm{G}$ then

$\sum_{u \in N[v]} g(u)=[(+1)+(-1)]+[(+1)+(-1)+(-1)]=-1$.

Sub case 2: Let $u_{\mathrm{i}} \notin P_{n}$ in $\mathrm{i}^{\text {th }}$ copy of $\mathrm{G}$ then

$\sum_{u \in N[v]} g(u)=[(+1)+(-1)]+[(-1)+(-1)+(-1)]=-3$.

(ii)As $\mathrm{d}(\mathrm{v})=3$ in $G$, then $\mathrm{N}[\mathrm{v}]$ contains 2 vertices of $C_{m}$ and two vertices of $P_{\mathrm{n}}$ in $G$.

Sub case 1: Let $u_{\mathrm{i}} \in P_{n}$ in $\mathrm{i}^{\text {th }}$ copy of $\mathrm{G}$ then

$\sum_{u \in N[v]} g(u)=[(+1)+(-1)]+[(-1)+(+1)]=0$.

Sub case 2: Let $u_{\mathrm{i}} \notin P_{n}$ in $\mathrm{i}^{\text {th }}$ copy of $\mathrm{G}$ then

$\sum_{u \in N[v]} g(u)=[(+1)+(-1)]+[(-1)+(-1)]=-2$.

Case 4: Suppose $\mathrm{v} \in C_{m}$ be such that $\mathrm{d}(\mathrm{v})=2$ in $G$,

(i)Here $\mathrm{N}[\mathrm{v}]$ contains 2 vertices of $C_{m}$ and one vertex of $P_{\mathrm{n}}$ in $G$ then $g(v)=-1$ or +1 .

Sub case 1: Let $u_{\mathrm{i}} \in P_{n}$ in $\mathrm{i}^{\text {th }}$ copy of $\mathrm{G}$.

If $g(v)=-1 \Rightarrow \sum_{u \in N[v]} g(u)=(-1)+[(-1)+(+1)]=-1$.

If $g(v)=+1 \Rightarrow \sum_{u \in N[v]} g(u)=(+1)+[(+1)+(-1)]=+1(>0)$.
Sub case 2: Let $u_{\mathrm{i}} \notin P_{n}$ in $\mathrm{i}^{\text {th }}$ copy of $\mathrm{G}$.

If $g(v)=-1 \Rightarrow \sum_{u \in N[v]} g(u)=(-1)+[(-1)+(-1)]=-3$.

If $g(v)=+1 \Rightarrow \sum_{u \in N[v]} g(u)=(+1)+[(-1)+(-1)]=-1$.

(ii)Here $\mathrm{N}[\mathrm{v}]$ contains 3 vertices of $C_{m}$ and zero vertex of $P_{\mathrm{n}}$ in $G$.

If $g(v)=-1 \Rightarrow \sum_{u \in N[v]} g(u)=(-1)+[(-1)+(+1)]=-1$.

If $g(v)=+1 \Rightarrow \sum_{u \in N[v]} g(u)=(+1)+[(-1)+(-1)]=-1$.

From the above cases, we get $\sum_{u \in N[v]} g(u)>0$, for some $v \in V$.

This implies that the function $g$ is not an inverse signed dominating function. Hence $\mathrm{f}$ is a maximal inverse signed dominating function on G. Now inverse signed total domination number is the sum of the function value of all vertices in $\mathrm{G}$, that is

$\sum_{u \in V(G)} f(u)=(\underbrace{\left\lfloor\frac{m}{3}\right\rfloor(+1)}_{n \text {-times }}+\underbrace{\left((m)-\left\lfloor\frac{m}{3}\right\rfloor\right)(-1)}_{n \text {-times }}=-m n+2 n\left\lfloor\frac{m}{3}\right\rfloor$.

Therefore $\gamma_{s}^{0}(G)=n\left[2\left\lfloor\frac{m}{3}\right\rfloor-m\right]$.

Case II: Suppose $\mathrm{m}=3 \mathrm{k}+2$

Let $f: V \rightarrow\{-1,+1\}$ be a function defined by

$f(v)=\left\{\begin{array}{l}+1, \text { if } m \equiv 1(\bmod 3) \text { vertices in each copy of } C_{m} \text { in } G, \\ -1, \quad \text { otherwise. }\end{array}\right.$

Here +1 is assigned to $\left\lceil\frac{m}{3}\right\rceil$ vertices in each copy of $C_{m}$ in $\mathrm{G}$,

-1 is assigned to all other vertices in $\mathrm{G}$.

Case 1: Suppose $\mathrm{v} \in P_{\mathrm{n}}$ be such that

(i) As $\mathrm{d}(\mathrm{v})=4$ in $G$ then

$\sum_{u \in N[v]} f(u)=[(+1)+(+1)]+[(-1)+(-1)+(-1)]=-1$.

(ii) As $d(v)=3$ in $G$ then

$\sum_{u \in N[v]} f(u)=[(+1)+(+1)]+[(-1)+(-1)]=0$.

Case 2: Suppose $\mathrm{v} \in C_{m}$ be such that $\mathrm{d}(\mathrm{v})=2$ in $G$.

(i) Here $\mathrm{N}[\mathrm{v}]$ contains 2 vertices of $C_{m}$ and one vertex of $P_{\mathrm{n}}$ in $G$ then $f(v)=+1$.

If $f(v)=+1 \Rightarrow \sum_{u \in N[v]} f(u)=(+1)+[(-1)+(-1)]=-1$.

(ii) Here $\mathrm{N}[\mathrm{v}]$ contains 3 vertices of $C_{m}$ and zero vertex of $P_{\mathrm{n}}$ in $G$ then $f(v)=-1$ and $f(v)=+1$.

If $f(v)=-1 \Rightarrow \sum_{u \in N[v]} f(u)=(-1)+[(-1)+(+1)]=-1$. 
If $f(v)=+1 \Rightarrow \sum_{u \in N[v]} f(u)=(+1)+[(-1)+(-1)]=-1$

From the above cases the function $f$ is an inverse signed dominating function, because $\sum_{u \in N[v]} f(u) \leq 0, \forall v \in V$.

Now maximality check for $f$, define $\mathrm{g}: \mathrm{V} \rightarrow\{-1,+1\}$ by

$g(v)=\left\{\begin{array}{l}+1, \text { if any one vertex } v=u_{i} \in P_{n} \text { in } G, \\ +1, \text { if }\left\lceil\frac{m}{3}\right\rceil \text { vertices in each copy of } C_{m} \text { in } G, \\ -1, \quad \text { otherwise. }\end{array}\right.$

Here two cases are followed.

Case 3: Suppose $v \in P_{\mathrm{n}}$ be such that

(i)As $\mathrm{d}(\mathrm{v})=4$ in $G$, then $\mathrm{N}[\mathrm{v}]$ contains 2 vertices of $C_{m}$ and three vertices of $P_{\mathrm{n}}$ in $G$.

Sub case 1: Let $u_{\mathrm{i}} \in P_{n}$ in $^{\text {th }}$ copy of $\mathrm{G}$ then

$\sum_{u \in N[v]} g(u)=[(+1)+(+1)]+[(-1)+(-1)+(+1)]=+1(>0)$.

Sub case 2: Let $u_{\mathrm{i}} \notin P_{n}$ in $\mathrm{i}^{\text {th }}$ copy of $\mathrm{G}$ then

$\sum_{u \in N[v]} g(u)=[(+1)+(+1)]+[(-1)+(-1)+(-1)]=-1$.

(ii)As $\mathrm{d}(\mathrm{v})=3$ in $G$, then $\mathrm{N}[\mathrm{v}]$ contains 2 vertices of $C_{m}$ and two vertices of $P_{\mathrm{n}}$ in $G$.

Sub case 1: Let $u_{\mathrm{i}} \in P_{n}$ in $\mathrm{i}^{\text {th }}$ copy of $\mathrm{G}$ then

$\sum_{u \in N[v]} g(u)=[(+1)+(+1)]+[(-1)+(+1)]=+2(>0)$.

Sub case 2: Let $u_{\mathrm{i}} \notin P_{n}$ in $\mathrm{i}^{\text {th }}$ copy of $\mathrm{G}$ then

$\sum_{u \in N[v]} g(u)=[(+1)+(+1)]+[(-1)+(-1)]=0$.

Case 4: Suppose $\mathrm{v} \in C_{m}$ be such that $\mathrm{d}(\mathrm{v})=2$ in $G$,

(i)Here $\mathrm{N}[\mathrm{v}]$ contains 2 vertices of $C_{m}$ and one vertex of $P_{\mathrm{n}}$ in $G$ then $g(v)=+1$.

Sub case (1): Let $u_{\mathrm{i}} \in P_{n}$ in $\mathrm{i}^{\text {th }}$ copy of $\mathrm{G}$ then

$\sum_{u \in N[v]} g(u)=(+1)+[(-1)+(-1)]=-1$.

Sub case (2): Let $u_{\mathrm{i}} \notin P_{n}$ in $\mathrm{i}^{\text {th }}$ copy of $\mathrm{G}$ then

$\sum_{u \in N[v]} g(u)=(+1)+[(-1)+(-1)]=-1$.

(ii)Then $\mathrm{N}[\mathrm{v}]$ contains 3 vertices of $C_{m}$ and zero vertex of $P_{\mathrm{n}}$ in $G$ then $g(v)=-1$ and $g(v)=+1$.

If $g(v)=-1 \Rightarrow \sum_{u \in N[v]} g(u)=(-1)+[(-1)+(+1)]=-1$.

If $g(v)=+1 \Rightarrow \sum_{u \in N[v]} g(u)=(+1)+[(-1)+(-1)]=-1$.

From the above cases, we get $\sum_{u \in N[v]} g(u)>0$, for some $v \in V$.

This implies that the function $g$ is not an inverse signed dominating function. Hence $f$ is a maximal inverse signed dominating function on G. Now inverse signed domination number is the sum of the function value of all vertices in G, that is

$$
\sum_{u \in V(G)} f(u)=(\underbrace{\left\lceil\frac{m}{3}\right\rceil(+1)}_{n-\text { times }})+\underbrace{\left((m)-\left\lceil\frac{m}{3}\right\rceil\right)(-1)}_{n-\text { times }}=-m n+2 n\left\lceil\frac{m}{3}\right\rceil .
$$

Therefore $\gamma_{s}^{0}(G)=n\left[2\left\lceil\frac{m}{3}\right\rceil-m\right]$.

\section{RESULTS ON CORONA PRODUCT GRAPH}

Theorem 3.1: A function $f: V \rightarrow\{-1,+1\}$ is defined by

$f\left(v_{i}\right)=\left\{\begin{array}{l}+1, \text { if } 1 \leq i \leq\left(\frac{m}{2}\right) \text { of each copy of } K_{m} \text { in } G, \\ -1, \text { otherwise. }\end{array}\right.$

is a maximal inverse signed dominating function of a graph $\mathrm{G}=P_{\mathrm{n}} \square K_{m}$ and inverse signed domination number is $\gamma_{s}^{0}(G)=-n$, if mis even.

Proof: Consider the graph $\mathrm{G}=P_{\mathrm{n}} \square K_{m}$ with $|V|$ number of vertices and $|E|$ number of edges.

Let $f$ be a function defined in the hypothesis.

Case 1: Let $\mathrm{v}_{i} \in P_{\mathrm{n}}$ be such that $\mathrm{d}\left(\mathrm{v}_{\mathrm{i}}\right)=\mathrm{m}+2$ in $G$ then

$$
\sum_{u \in N\left[v_{i}\right]} f(u)=(-1)+(-1)+(-1)+\left[\left(\frac{m}{2}\right)(-1)+\left(\frac{m}{2}\right)(+1)\right]=-3 .
$$

Case 2: Let $\mathrm{v}_{i} \in P_{\mathrm{n}}$ be such that $\mathrm{d}\left(\mathrm{v}_{\mathrm{i}}\right)=\mathrm{m}+1$ in $G$ then

$$
\sum_{u \in N\left[v_{i}\right]} f(u)=(-1)+(-1)+\left[\left(\frac{m}{2}\right)(-1)+\left(\frac{m}{2}\right)(+1)\right]=-2 \text {. }
$$

Case 3: Let $\mathrm{v}_{i} \in K_{m}$ be such that $\mathrm{d}\left(\mathrm{v}_{\mathrm{i}}\right)=m$ in $G$ and $\mathrm{f}\left(\mathrm{v}_{\mathrm{i}}\right)=-1$ or +1 .

If

$\mathrm{f}\left(\mathrm{v}_{\mathrm{i}}\right)= \pm 1 \Rightarrow \sum_{u \in N\left[v_{i}\right]} f(u)=(-1)+\left[\left(\frac{m}{2}\right)(-1)+\left(\frac{m}{2}\right)(+1)\right]=-1$.

Hence for all the above possibilities, we get $\sum_{u \in N\left[v_{i}\right]} f(u)<0, \forall v_{i} \in V$. This implies that the function $f$ is an inverse signed dominating function. Now the maximality check for $f$, define $\mathrm{g}: \mathrm{V} \rightarrow\{-1,+1\}$ by

$$
g\left(v_{i}\right)= \begin{cases}+1, & \text { if } 1 \leq i \leq \frac{m}{2} \text { of each copy of } K_{m} \text { in } G, \\ +1, & \text { if } v_{i}=v_{k} \in P_{n} \text { in } G \\ -1, & \text { otherwise. }\end{cases}
$$

Case 1: Let $\mathrm{v}_{i} \in P_{\mathrm{n}}$ be such that $\mathrm{d}\left(\mathrm{v}_{\mathrm{i}}\right)=\mathrm{m}+2$ in $G$. 
(i) If $v_{k} \in N\left[v_{i}\right] \Rightarrow \sum_{u \in N\left[v_{i}\right]} g(u)=1+(-1)+(-1)+\left[\left(\frac{m}{2}\right)(+1)+\left(\frac{m}{2}\right)(-1)\right]=-1$.
(ii)If $v_{k} \notin N\left[v_{i}\right] \Rightarrow \sum_{u \in N\left[v_{i}\right]} g(u)=(-1)+(-1)+(-1)+\left[\left(\frac{m}{2}\right)(+1)+\left(\frac{m}{2}\right)(-1)\right]=-3$.

Case 2: Let $\mathrm{v}_{i} \in P_{\mathrm{n}}$ be such that $\mathrm{d}\left(\mathrm{v}_{\mathrm{i}}\right)=\mathrm{m}+1$ in $G$.

(i) If $v_{k} \in N\left[v_{i}\right] \Rightarrow \sum_{u \in N\left[v_{i}\right]} g(u)=1+(-1)+\left[\left(\frac{m}{2}\right)(+1)+\left(\frac{m}{2}\right)(-1)\right]=0$.

(ii)If $v_{k} \notin N\left[v_{i}\right] \Rightarrow \sum_{u \in N\left[v_{i}\right]} g(u)=(-1)+(-1)+\left[\left(\frac{m}{2}\right)(+1)+\left(\frac{m}{2}\right)(-1)\right]=-2$.

Case 3: Let $\mathrm{v}_{i} \in K_{m}$ be such that $\mathrm{d}\left(\mathrm{v}_{\mathrm{i}}\right)=m$ in $G$ and $g\left(v_{i}\right)= \pm 1$.

(i) Let $\mathrm{v}_{k} \in N\left[v_{i}\right] \Rightarrow \sum_{u \in N\left[v_{i}\right]} g(u)=1+\left[\left(\frac{m}{2}\right)(+1)+\left(\frac{m}{2}\right)(-1)\right]=1$.

(ii) Let $\mathrm{v}_{k} \notin N\left[v_{i}\right] \Rightarrow \sum_{u \in N\left[v_{i}\right]} g(u)=(-1)+\left[\left(\frac{m}{2}\right)(+1)+\left(\frac{m}{2}\right)(-1)\right]=-1$.

This implies that $\mathrm{g}$ is not an inverse signed dominating function because $\sum_{u \in N\left[v_{i}\right]} g(u)>0$, for some $v_{i} \in V$.

Hence $f$ is a maximal inverse signed dominating function on $G$. Now inverse signed domination number is the sum of the function value of all vertices in $G$, that is

$\sum_{u \in V(G)} f(u)=\underbrace{(-1)+---+(-1)}_{n \text {-times }}+[\underbrace{\left(\frac{m}{2}\right)(+1)+\left(\frac{m}{2}\right)(-1)}_{n \text {-times }}]=-n$.

Finally $\gamma_{s}^{0}(G)=-n$, if $m$ is even.

Theorem 3.2: A function $f: V \rightarrow\{-1,+1\}$ is defined by

$f\left(v_{i}\right)= \begin{cases}+1, & \text { if } 1 \leq i \leq\left(\frac{m+1}{2}\right) \text { of each copy of } K_{m} \text { in } G, \\ -1, & \text { otherwise. }\end{cases}$

is a maximal inverse signed dominating function of a graph $\mathrm{G}=P_{\mathrm{n}} \square K_{m}$ and inverse signed domination number is $\gamma_{S}^{0}(G)=0$, if $\mathrm{m}$ is odd.

Proof: Let $f$ be a function defined in the hypothesis.

Case 1: Let $\mathrm{v}_{i} \in P_{\mathrm{n}}$ be such that $\mathrm{d}\left(\mathrm{v}_{\mathrm{i}}\right)=\mathrm{m}+2$ in $G$ then

$$
\sum_{u \in N\left[v_{i}\right]} f(u)=(-1)+(-1)+(-1)+\left[\left(\frac{m+1}{2}\right)(+1)+\left(\frac{m-1}{2}\right)(-1)\right]=-2 .
$$

Case 2: Let $\mathrm{v}_{i} \in P_{\mathrm{n}}$ be such that $\mathrm{d}\left(\mathrm{v}_{\mathrm{i}}\right)=\mathrm{m}+1$ in $G$ then

$$
\sum_{u \in N\left[v_{i}\right]} f(u)=(-1)+(-1)+\left[\left(\frac{m+1}{2}\right)(+1)+\left(\frac{m-1}{2}\right)(-1)\right]=-1 \text {. }
$$

Case 3: Let $\mathrm{v}_{i} \in K_{m}$ be such that $\mathrm{d}\left(\mathrm{v}_{\mathrm{i}}\right)=m$ in $G$ and $\mathrm{f}\left(\mathrm{v}_{\mathrm{i}}\right)= \pm 1 \Rightarrow \sum_{u \in N\left[v_{i}\right]} f(u)=(-1)+\left[\left(\frac{m+1}{2}\right)(+1)+\left(\frac{m-1}{2}\right)(-1)\right]=0$.

Hence for all the above possibilities, we get

$$
\sum_{u \in N\left[v_{i}\right]} f(u) \leq 0, \forall v_{i} \in V .
$$

This implies that the function $f$ is an inverse signed dominating function.

Now the maximality check for $f$, define $\mathrm{g}: \mathrm{V} \rightarrow\{-1,+1\}$ by

$$
g\left(v_{i}\right)= \begin{cases}+1, & \text { if } 1 \leq i \leq \frac{m+1}{2} \text { of each copy of } K_{m} \text { in } G, \\ +1, & \text { if } v_{i}=v_{k} \in P_{n} \text { in } G \\ -1, & \text { otherwise. }\end{cases}
$$

Case 1: Let $\mathrm{v}_{i} \in P_{\mathrm{n}}$ be such that $\mathrm{d}\left(\mathrm{v}_{\mathrm{i}}\right)=\mathrm{m}+2$ in $G$.

$$
\begin{aligned}
& \text { (i) If } v_{k} \in N\left[v_{i}\right] \Rightarrow \sum_{u \in N\left[v_{i}\right]} g(u)=1+(-1)+(-1)+\left[\left(\frac{m-1}{2}\right)(-1)+\left(\frac{m+1}{2}\right)(+1)\right]=0 . \\
& \text { (ii) If } v_{k} \notin N\left[v_{i}\right] \Rightarrow \sum_{u \in N\left[v_{i}\right]} g(u)=(-1)+(-1)+(-1)+\left[\left(\frac{m-1}{2}\right)(-1)+\left(\frac{m+1}{2}\right)(+1)\right]=-2 .
\end{aligned}
$$

Case 2: Let $\mathrm{v}_{i} \in P_{\mathrm{n}}$ be such that $\mathrm{d}\left(\mathrm{v}_{\mathrm{i}}\right)=\mathrm{m}+1$ in $G$.

(i) If $v_{k} \in N\left[v_{i}\right] \Rightarrow \sum_{u \in N\left[v_{i}\right]} g(u)=1+(-1)+\left[\left(\frac{m-1}{2}\right)(-1)+\left(\frac{m+1}{2}\right)(+1)\right]=1$.

(ii) If $v_{k} \notin N\left[v_{i}\right] \Rightarrow \sum_{u \in N\left[v_{i}\right]} g(u)=(-1)+(-1)+\left[\left(\frac{m-1}{2}\right)(+1)+\left(\frac{m+1}{2}\right)(-1)\right]=-3$.

Case (3): Let $\mathrm{v}_{i} \in K_{m}$ be such that $\mathrm{d}\left(\mathrm{v}_{\mathrm{i}}\right)=m$ in $G$ and $g\left(v_{i}\right)= \pm 1$.

(i) Let $v_{k} \in N\left[v_{i}\right] \Rightarrow \sum_{u \in N\left[v_{i}\right]} g(u)=1+\left[\left(\frac{m-1}{2}\right)(-1)+\left(\frac{m+1}{2}\right)(+1)\right]=2$

(ii) Let $v_{k} \notin N\left[v_{i}\right] \Rightarrow \sum_{u \in N\left[v_{i}\right]} g(u)=(-1)+\left[\left(\frac{m-1}{2}\right)(-1)+\left(\frac{m+1}{2}\right)(+1)\right]=0$.

This implies that $g$ is not an inverse signed dominating function because $\sum_{u \in N\left[v_{i}\right]} g(u)>0$, for some $v_{i} \in V$.

Hence $f$ is a maximal inverse signed dominating function on $G$. Now inverse signed domination number is the sum of the function value of all vertices in $G$, that is

$$
\sum_{u \in V(G)} f(u)=\underbrace{(-1)+---+(-1)}_{n \text {-times }}+[\underbrace{\left(\frac{m+1}{2}\right)(+1)+\left(\frac{m-1}{2}\right)(-1)}_{n \text {-times }}]=0
$$

Finally $\gamma_{s}^{0}(G)=0$, if $m$ is odd.

\section{CONCLUSION}

It is interesting to study the inverse signed dominating functions of corona product graph of complete graph with a path and rooted product graph of a path with cycle. This work gives the scope for an extensive study of various inverse dominating functions of these graphs. 


\section{REFERENCES}

[1] C. D. Godsil, and B. D. McKay, "A new graph product and its spectrum", Bulletin of the Australian Mathematical Society, 18(01), (1978), 21-28.

[2] C. Lu, S. L. Peng, and C. Tang, "Efficient minus and signed domination in graphs", Algorithms and Computation, (2000), 267-269.

[3] H. U. A. N. G. Zhong-sheng, "On Inverse Signed Total Domination in Graphs", Journal of Langfang Teachers College, (Natural Science Edition), 3, (2010).
[4] J. E. Dunbar, S. T. Hedetniemi, M. A. Henning and P. J. Slater, "Signed Domination in Graphs, Graph Theory", Combinatorics and Application, 1, (1995), 311-322.

[5] O. Favaron, "Signed domination in regular graphs", Discrete Math., 158, (1996), 287-293.

[6] R. Frucht and F. Harary, "On the corona of Two Graphs", Aequationes Mathematicae, 4, (1970), 322 - 325.

[7] Z. Furedi, D. Mubayi, "Signed domination in regular graphs and set systems”, J. Combin. Theory B, 76, (1999), 223-239. 